\title{
Depth-of-Field Blur Effects for First-Person Navigation in Virtual Environments
}

\author{
Sébastien Hillaire* \\ Univ. of Rennes 1 / IRISA \\ Anatole Lécuyer ${ }^{\dagger}$ \\ INRIA / IRISA
}

\author{
Rémi Cozot ${ }^{\ddagger}$ \\ Univ. of Rennes 1 / IRISA
}

\author{
Géry Casiez ${ }^{\S}$ \\ Univ. of Lille 1 / INRIA
}

\begin{abstract}
This paper studies the use of visual blur effects, i.e., blurring of parts of the image fed back to the user, for First-Person-Navigations in Virtual Environments (VE). First, we introduce a model of dynamic visual blur for VE which is based on two types of blur effect: (1) a Depth-of-Field blur (DoF blur) which simulates the blurring of objects located in front or back of the focus point of the eyes, and (2) a peripheral blur which simulates the blurring of objects located at the periphery of the field of vision. We introduce two new techniques to improve real-time DoF: (1) a paradigm to compute automatically the focal distance, and (2) a temporal filtering that simulates the accommodation phenomenon. Second, we describe the results of a pilot experiment conducted to study the influence of blur effects on the performance and preference of video gamers during multiplayer sessions. Interestingly, it seems that visual blur effects did not degrade performance of gamers and they were preferred and selected by nearly half of the participants to improve fun and game-play. Taken together, our results suggest that the use of visual blur effects could thus be suitable in videogames and in other virtual environments.
\end{abstract}

CR Categories: H.1.2 [Information Systems]: User/Machine Systems-Human factors H.5.1 [Information Interfaces and Presentation]: Multimedia Information Systems-Artificial, augmented, and virtual realities H.5.2 [Information Interfaces and Presentation]: User Interfaces-Input devices and strategies, Interaction styles, User-centred design

Keywords: visual blur, depth-of-field blur, peripheral blur, focalization, accommodation, first-person-navigation, videogames

\section{Introduction}

Depth-of-field (DoF) of the human's eyes is the range of distances near the point of focus where the eyes perceive the image as sharp. Objects behind and in front of the point of focus are blurred. DoF and its associated blur effects are well-known and classical depth cues in human vision [Atchinson and Smith 2000]. Without a DoF blur, virtual images may sometimes look "too synthetic" or "too perfect". Visual blur effects have not been introduced into the Virtual Reality applications yet and, as for today, there is a need for two kinds of investigation about the use of visual blur in Virtual Environments: (1) development of new models of realistic visual blur effects for VE that take into account interactivity and real-time con-

\footnotetext{
*e-mail: shillair@irisa.fr

†e-mail: anatole.lecuyer@irisa.fr

‡e-mail: cozot@irisa.fr

$\S$ e-mail: gery.casiez@lifl.fr
}

Copyright $\odot 2007$ by the Association for Computing Machinery, Inc.

Permission to make digital or hard copies of part or all of this work for personal or classroom use is granted without fee provided that copies are not made or distributed for commercial advantage and that copies bear this notice and the full citation on the first page. Copyrights for components of this work owned by others than ACM must be honored. Abstracting with credit is permitted. To copy otherwise, to republish, to post on servers, or to redistribute to lists, requires prior specific permission and/or a fee. Request permissions from Permissions Dept, ACM Inc., fax +1 (212) 869-0481 or e-mail permissions@acm.org.

VRST 2007, Newport Beach, Califomia, November 5-7, 2007.

(c) 2007 ACM 978-1-59593-863-3/07/0011 $\$ 5.00$ straint and (2) evaluation of the use of visual blur effects in terms of both performance and subjective preference in VE. In the remainder of this paper, we focus our study on situations of first-person navigations in virtual environments with a relatively small field of view. After describing briefly related work in the field of visual blur effects used in computer graphics, we introduce a model of dynamic visual blur for first-person navigation in virtual environments. Then, we report on an experiment conducted to study the influence of visual blur on performance and preference of gamers during multiplayer sessions of a first-person-shooter game.

\section{Related work}

The simulation of visual blur was introduced early in Computer Graphics to improve the photorealistic aspect of synthetic images. Potmesil and Chakravarty [1981] were the first to propose the simulation of an optical lens to simulate depth-of-field blur. The lens simulation provides the amount of blur for each pixel according to its depth: a point which is out of focus becomes a disk or circle after the projection through the lens. The resulting circle is called the Circle of Confusion (CoC). The diameter of this circle of confusion corresponds to the amount of blur [Potmesil and Chakravarty 1981]. After this pioneer study, most researchers used this algorithm [Demers 2004]. Later Barsky [2004] introduced the alternative concept of vision-realistic rendering that accurately simulate the image perceived by the eye. Other visual blur than DoF blur can be used to enhance the visual appearance of digital images, such as the peripheral blur [Anstis 1998] which refers to the coarser acuity of the eyes from the fovea to the periphery and the motion blurs [Max and Lerner 1985] wich correspond to the filming of objects moving rapidly.

To the author's best knowledge, the use of visual blur effects in real-time applications of virtual reality has been suggested (but not implemented) by Rokita [1996]. He stated that such blur effect was "especially important in VR applications". Rokita recommended the use of an eye tracking system to compute the point of focus. In videogames, attempts to implement blur effects in real-time have been achieved recently. The Unreal game engine version 2 .X proposes DoF blur together with motion blur for the latest generation of games, such as "Call of Juarez" (developer: Techland, publisher: Focus home entertainment, September 2006). In this videogame, the DoF blur remains however limited to a "sniper mode" with few depth plans.

\section{Visual blur effects for first-person naviga- tion in virtual environments}

In this section we describe a model of dynamic visual blur for firstperson navigations in VE. It is based on the combination of two blur effects: (1) a depth-of-field blur and (2) a peripheral blur.

\subsection{Depth-of-Field blur effect}

The Depth-of-Field blur effect simulates the visual blurring of pixels of objects located in front or back of the focus point. The focus point is associated with a focal distance (fd), i.e., the distance between the eyes (or camera) and the focus point. 


\subsubsection{Use of a lens model}

We use here the classical model of the lens [Potmesil and Chakravarty 1981]. In this model, the amount of blur, i.e., the diameter of the circle of confusion $D C o C d$ of a point projected on screen is given by Equation 1, in which $D$ is the lens diameter, $f$ the focal length of the lens, $f_{d}$ is the focal distance and $z$ the depth of the point.

$$
D C o C_{d}=\left|\frac{D \times f \times\left(f_{d}-z\right)}{f_{d} \times(z-f)}\right|
$$

\subsubsection{Automatic computation of the focal distance}

An optimal way to determine in real-time the focal distance would be to use an eye-tracking system. However, such devices are still expensive, complex and not available on a mass market. Thus, in absence of an eye-tracking system, we propose to use a paradigm used in First-Person-Shooter games (FPS). In FPS games, the user manipulates with his/her 2D mouse and keyboard a virtual visor always located at the centre of the screen. In this case, we can assume that the user mainly looks at the part of the screen close to the visor. Actually, using an eye-tracking system, Kenny et al. [2005] found that gamers of FPS videogames watched indeed more than $82 \%$ of the time the central area of the monitor. Then, we introduce the notion of "focus area", i.e., a square area located at the centre of the screen that the user is supposed to preferentially look at. This focus area recalls auto-focus systems used in digital camera which also aim at providing an appropriate focal distance when taking a picture (see Figure 1).
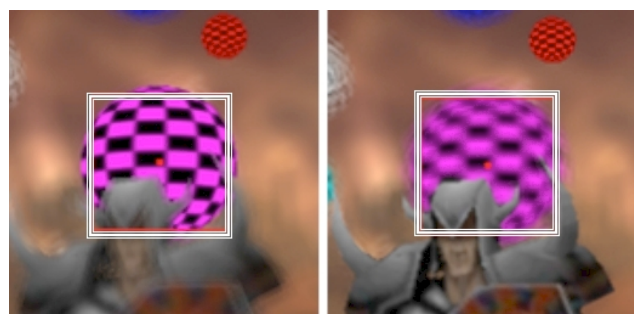

Figure 1: Depth-of-Field Blur when using a focus area (white square), without (left) and with (right) semantic weighting.

The depth of each pixel of the focus area is first computed using an auxiliary buffer. Then, the choice of the function used to compute the focal distance from all the depths of the pixels enclosed in the focus area is very important. Minimum, Maximum, and Average functions are good candidates. In the case of an FPS game, some objects of the environment are known to be more important than others (e.g., enemies or bonus-objects). For this aim, we propose to use a semantic weighting of the pixels. The semantic weighting is done to increase the weight of pixels corresponding to objects (or targets) that are known to be important in the simulation. To do so, we can add to the initial description of the virtual objects a field corresponding to its visual semantic weight. The semantic weight of each pixel varies between $W S \min$ and $W S \max$. Figure 1 illustrates the use of semantic weighting. In this example, the weight of the character/enemy in front is much higher than the one of the decorative spheres located behind. Even if the character covers fewer pixels (less than one quarter of the area), the focus is done systematically on it.

In addition, a spatial weighting modifies slightly the weight of the central pixels. It is achieved by using a Gaussian function that gives a weight of WGmax to the centre and WGmin to the borders of the area. Finally, the resulting focal distance is computed using Equation 2, with $W S(p)$ the semantic weight of pixel $p, W G(x)$ the Gaussian spatial weight for distance $x, d 2 A C(p)$ the distance of $p$ to the center of the focus area. In our final implementation, edge of the focus area was set to 33 pixels (i.e., $11.9 \mathrm{~mm}$ on a 17 ' screen with a resolution of $1024 \times 768$ pixels), WGmin was set to 0.7,WGmax to 1,WSmin to 0.004 and $W S \max$ to 1 .

$$
f_{d}=\frac{\sum_{p \in \text { area }} W S(p) \times W G(d 2 A C(p)) \times \operatorname{depth}(p)}{\sum_{p \in \text { area }} W S(p) \times W G(d 2 A C(p))}
$$

\subsubsection{Simulation of accommodation phenomenon}

In the human visual system, the accommodation of our eyes when changing the focus point takes few milliseconds. Thus, we propose to simulate the phenomenon of accommodation within our DoF blur effect. To do so, we add a temporal filter to the computation of the final focal distance. After preliminary testing, we chose a lowpass filtering, given by Equation 3, with $\tau=(\pi \times f c) / 2, f c$ the cut-off frequency, $T e$ the sampling period, $\overline{f_{d}}(n)$ the filtered focal distance at frame $\mathrm{n}$, and $f_{d}(n)$ the focal distance given by the autofocus system (before filtering). In our final implementation, we used $T e=1 / 70$, and $f c=5 \mathrm{~Hz}$.

$$
\overline{f_{d}}(n)=\left(f_{d}(n)+\frac{\tau}{T e} \overline{f_{d}}(n-1)\right) \frac{1}{1+\frac{\tau}{T e}}
$$

\subsection{Peripheral blur effect}

The peripheral blur effect simulates the fact that sharpness of objects perceived visually decreases when these objects are located at the periphery of the human field of vision. Thus, this effect blurs progressively the pixels that are located at a certain distance from the centre of the focus area. It is independent of the depth-of-field. The peripheral blur is added here as a supplementary visual effect expected to improve the sensations of the user. However, the main objective of the peripheral blur effect is also to incite the user to look at the centre of the screen. Indeed, by blurring the contour of the image, we hope the user will be implicitly forced to look through the visor, i.e., inside the focus area. The computation of the amount of peripheral blur for each pixel (diameter of Circle of Confusion $D C o C_{p}$ ) is given by Equation 4 , in which $\mathbf{z}$ is the lookat direction (e.g., direction of the camera, in the case when the focus area is at the centre of the image) and $\mathbf{p}$ is the normalised direction of the pixel in the camera frame. In our final implementation, we used a power $n$ equal to 2 .

$$
{ }^{D C o} C_{p}=\left(\sqrt{\frac{1}{\mathbf{z} \cdot \mathbf{p}}-1}\right)^{n}
$$

\subsection{Final visual blur computation}

Once the computation of both the peripheral and the DoF blur is achieved, we can compute the total amount of blur for each pixel, i.e., the final diameter of its circle of confusion $D C o C_{f}$. We use the normalized contributions of both the peripheral and DoF blurs as follows:

$$
D C o C_{f}=D_{\max } \times \min \left(1, D C o C_{d}+D C o C_{p}\right)
$$

with Dmax the maximum amount of blur (maximum diameter of the pixel's final $\mathrm{CoC}$ ), $\mathrm{DCoC}_{d}$ the normalized diameter of the DoF 
blur's $\mathrm{CoC}$ and $D C o C_{p}$ the normalized diameter of the peripheral blur's CoC. In our implementation $D_{\max }=12$ pixels.

The blur can then be applied to the image by mixing each pixel's color with the colors of the pixels that are located inside its circle of confusion, according to a Poisson disk distribution. The elimination of the color leaking artifacts is achieved by using the gathering technique [Demers 2004]. These effects were implemented successfully in the open engine of the Quake III videogame [IdSoftware 2007] (see Figure 2).

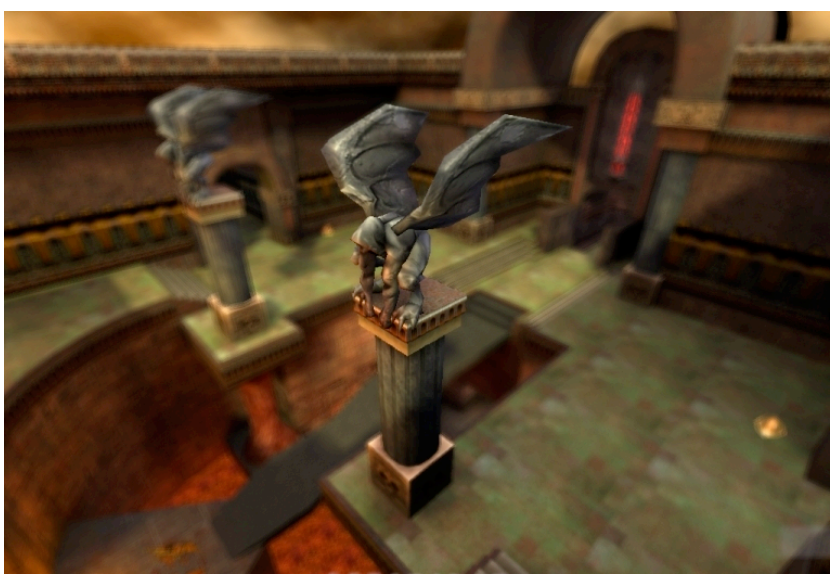

Figure 2: Quake III videogame with blur effects implemented.

\section{Pilot experiment}

A pilot experiment has been conducted to study the influence of our visual blur effects on the performance and subjective preference of gamers. Prior to the experiment we could formulate the two following hypotheses: (H1) visual blur could degrade performance of participants (since the visual feedback and the displayed image is blurred to some extent), and (H2) visual blur could improve fun (since it provides a supplementary visual effect and it potentially increases the realism of the visual feedback).

\subsection{Experimental apparatus}

The visual blur effects were all implemented inside the 3D engine of Quake III, i.e., both the peripheral blur and the DoF blur with automatic focal distance computation, and accommodation simulation. Both the semantic and spatial weights were activated. The semantic weights of enemies were set to WSmax and all other semantic weights were set to WSmin. Other numerical values used in our implementation are given in the previous sections. The Quake III map used in our experiment was the map labelled "Q3DM7". We used 8 identical PCs on a local network with the same graphics cards connected to same monitors with same resolution. There was no perceivable lag. Participants were all provided with infra-red mouse and stereo headset for audio feedback.

\subsection{Experimental procedure}

The task consisted in playing a first-person-shooter game in deathmatch mode (each player was fighting against all the other players). Participants were instructed to be as precise as possible when shooting while using as little ammunition as possible. To reduce the variability across subjects, all players had only one weapon (a submachine gun) with unlimited ammunition. All life packs or special bonus were also removed from the map. The life was increased to 200 points (normal situation: 100 points) to increase the duration of the fights.

32 subjects ( 29 males, 3 females) with a mean age of $25.3(\mathrm{SD}=5.5)$ participated in our experiment.

A within-subjects design was used. The independent variable was the visual effect (no blur effects vs. blur effects). The experiment lasted 120 minutes including breaks. The participants were randomly divided into 4 groups of 8 subjects each. The experiment was then divided into two parts. The first part consisted in a training session with or without blur followed by the real experiment with a first session of 12 minutes in the same conditions as the training session and a second 12 minutes session with the reverse condition. Participants were asked to fill out a subjective questionnaire after these two sessions. The second part consisted in a performance test including 6 sessions of 10 minutes each. For each of the 6 sessions, 4 subjects played with blur and the other 4 subjects played without blur. The blur condition was automatically swapped for all players when running a new session. At the end, a general appreciation questionnaire was filled by all subjects. In each part of the experiment, the order of presentation was counterbalanced against participants and groups.

\subsection{Results}

\subsubsection{Performance}

The dependant variables were recorded for each participant at the end of each of the 6 sessions of the performance test. These performance variables were: the number of enemies that the player could kill during the session (frags), the number of times the player died (deaths), the total number of his/her shoots (total shoots), and his/her precision (precision, in percent) which is computed as the ratio between the number of succeeded shoots and the total number of shoots.

Interestingly, the performance of the players when they were exposed to the visual blur seems similar to their performance when the blur effect was not activated (see Table 1). The mean and standard deviation for each of the dependant variables are actually very close.

A repeated measures analysis of variance (ANOVA) was performed on the data. The ANOVA first showed that the presentation order of the visual blur effects and groups had no significant effect on the different dependant variables indicating that a within-subjects design was appropriate. Then, no significant main effect for visual blur effect was found for the different dependant variables. A post hoc statistical power analysis showed that our analysis has a power above $80 \%$ to detect a large size effect (effect size $=0.4$ ). This is sufficient to conclude that there is no large effect size on our results. Thus, our results tend to show that participants could fortunately maintain a similar level of performance, and we found no large degradation of performance with the activation of the blur effects.

\begin{tabular}{|c|c|c|c|c|}
\cline { 2 - 5 } \multicolumn{1}{c|}{} & \multicolumn{2}{c|}{ No blur effects } & \multicolumn{2}{c|}{ Blur effects } \\
\cline { 2 - 5 } \multicolumn{1}{c|}{} & Mean & SD & Mean & SD \\
\hline Frags & 28.7 & 9.9 & 28.5 & 10.0 \\
\hline Death & 28.6 & 4.7 & 28.7 & 4.6 \\
\hline Total shoots & 2694 & 496 & 2653 & 509 \\
\hline Precision (\%) & 29.2 & 5.3 & 29.1 & 5.5 \\
\hline
\end{tabular}

Table 1: Mean and Standard Deviation (SD) for each dependant variables of the performance test, with and without the blur effects. 


\subsubsection{Questionnaire and user feedback}

After the two first sessions of 15 minutes, a large majority of people (84\% i.e., 27 participants out of 32) were able to notice a difference between the sessions. Then, 78\% (25/32) were able to formulate explicitly that a blur effect was applied to the image. Some participants could not clearly understand the meaning and/or computation of this blur effect: "strange blurring effect", "unsystematic blur", "intermittent blur".

The final and general appreciation questionnaire did not show a significant trend concerning a potential appreciation or detestation of the blur effects. Indeed, participants were balanced concerning: the improvement of realism of the virtual scene (14 preferred with the blur, 16 preferred without, and 2 had no preference), the improvement of fun (13 with, 13 without, 6 no preference), the improvement of perception of depth and distances in the VE (16 with, 13 without, 3 no preference) and the improvement of feeling of presence (11 with, 11 without, 10 no preference).

The people who preferred the game when the blur effects were activated could be very enthusiastic: "I was more intense with the blur", "it becomes addictive", "it is difficult to come back to the no-blur situation", "it is more fun", "more realistic", "the gameplay is improved", "the game is more credible". It seems thus that some participants would be ready to select and activate the blur effects for an in-game use.

The people who preferred the game without the blur effects generally felt tired (mainly eyes or headache). They estimated that the blurring effect was responsible for their fatigue $(31 \%, 10 / 32)$. Some of them, and more especially some expert gamers, found that the blur was a "discomfort". They perceived the blur as "too strong", "especially on the borders". Furthermore, these persons were annoyed by the blur when exploring the image and looking for targets/enemies on the screen.

Meanwhile, a majority of participants $84 \%$ (27/32) declared that the presence of the blur did not modify their strategy when fighting in the game.

\subsection{Discussion}

Our results concerning the constant performance of participants in both conditions fortunately contradict our first hypothesis H1. This seems consistent with the fact that participants subjectively perceived that their strategy was similar with or without the blur effects. Indeed, since the participants did not change their strategy, they could maintain the same level of performance through all the sessions. Our second hypothesis $\mathrm{H} 2$ was that blur effects could improve subjective preference concerning factors like fun of the game. It is confirmed by the fact that nearly half of players preferred the presence of blur in terms of fun, presence, or realism of the virtual environment. This seems sufficient for these blur effects to be recommended, for instance in videogames. But we also noticed that the same number of participants disliked the effects during the sessions, although it did not seem to degrade their performance.

The comments of the several participants suggest that the participants did not look constantly inside the focus area, i.e., at the centre of the screen. There were at least two phases that can be stressed. In one phase, the player shot at the enemies with his/her visor. In that case, the focal distance computed was always well adapted to the user's gaze. In a second phase, the player explored the image to find and identify the enemies. During this phase, the focal distance computed by our model might not correspond to the actual attention of the user. For some users, this situation was problematic and it generated both a discomfort and a fatigue. However, this visual fatigue did not prevent them from keeping their level of performance constant. For other users, this situation was apparently not a problem and the game could still look more credible or more fun.

During our experimental evaluation, we had voluntarily strengthened the blur, for the participants to better perceive its effects. However, several participants complained that the blurring was too strong. This might explain the fatigue observed and expressed by some of them. It suggests also that the intensity of the blur effects should be carefully tuned, or that the user should have the possibility to change the level of blur in his/her application.

\section{Conclusion}

We have studied the use of visual blur effects for first-person navigations in virtual environments. A model of dynamic visual blur was introduced which is based on two types of blur effect: a depthof-field blur which simulates the blurring of objects related to focalization, and a peripheral blur that simulates the blurring of the periphery of the field of view. To make the DoF blur effect more suitable in VR applications, we have developed both a technique to compute the focal distance automatically, together with a temporal filtering of the focal distance to simulate the phenomenon of accommodation. Then, a pilot experiment was conducted which showed that the activation of our visual blur effects did not seem to impair performance of gamers during multiple sessions of a multiplayer FPS game. Furthermore, nearly half of the participants preferred when the blur effects were activated, as it increased the realism of the visual feedback or simply for fun. Taken together, our results suggest that using blur effects could be suitable in videogames and in other VE.

\section{References}

Anstis, S. 1998. Picturing peripheral acuity. Perception 27, 817825 .

Atchinson, D., And Smith, G. 2000. Optics of the Human Eye. Elsevier Health Science.

BARSKY, B. A. 2004. Vision-realistic rendering: Simulation of the scanned foveal image from wavefront data of human subjects. Proceedings of Symposium on Applied Perception in Graphics and Visualization, 73-81.

DEMERS, J. 2004. Depth of field: A survey of techniques. In GPU Gems, Addison-Wesley Professional, 375-390.

IDSOFTwARE, 2007. http://www.idsoftware.com/.

Kenny, A., Delaney, H., Mcloone, S., And Ward, T. 2005. A preliminary investigation into eye gaze data in a first person shooter game. In Proceedings of European Conference on Modelling and Simulation, Addison-Wesley Professional.

MAX, N., AND LERNER, D. 1985. A two-and-a-half-d motionblur algorithm. In Proceedings of ACM SIGGRAPH, vol. 19, 85-93.

Potmesil, M., AND ChaKRAVARTy, I. 1981. A lens and aperture camera model for synthetic image generation. In Proceedings of ACM SIGGRAPH, vol. 19, 298-306.

RoKITA, P. 1996. Generating depth-of-field effects in virtual reality applications. In Proceedings of IEEE Computer Graphics and Applications, vol. 16, 18-21. 